\title{
Post-traumatic stress disorder in Israeli children
}

\author{
Sam Tyano
}

Geha Psychiatric Hospital, PO Box 102, Petach-Tikvah, Israel, email styano@post.tau.ac.il

nfortunately, terrorism, violence and other acute adverse life events have become a world-wide problem. There is no country today that is protected from these phenomena and people can no longer feel safe anywhere. This new situation has increased both the scientific interest in post-traumatic stress disorder (PTSD) and the amount of research conducted on this issue.

Currently, PTSD is studied in three ways:

o as a syndrome, which has to be well defined in order to be accurately diagnosed

O as a debilitating disorder which has harmful effects on people's well-being, and as such has to be cured

- as a human reaction to various stresfful events, whose risk and protective factors have to be studied in an effort to elaborate means of prevention.

Although an increasing amount of research into PTSD had been conducted in the past few years, there is still much to be done. The international scientific community needs to agree well defined criteria for its diagnosis, to formulate reliable and valid scales of measurement, and to plan methods of treatment. The present lack of agreed definitions and measurement tools makes it hard, and at times impossible, to compare epidemiological research results from around the world.

The difficulty in comparing and implementing research results is compounded by the fact that PTSD, to a very large extent, is a disorder that is determined by environmental factors. Although psychiatrists are trained to deal solely with individuals, when we come to determine the severity of this psychopathology, we must take into consideration the specific environment to which the subject was exposed. Hence, we must bear in mind that the groups studied will have experienced different types of stressor and different degrees and length of exposure.

A third problem concerns the symptoms or criteria required for the diagnosis of PTSD. When we use the terms 'acute' or 'chronic' PTSD, we mean a full-blown clinical picture. $N$ evertheless, recent publications have stressed the impact of:

O sub-threshold clinical PTSD symptoms and their implications for the development of a full clinical picture

o partial PTSD, which can have a profound effect on the development of future impairments.

In the preceding thematic paper in this issue on PTSD in children, Panos Vostanis discusses the relationship between the intensity of stresso rs and the epidemiology of PTSD among children in the GazaStrip. This important and pertinent article summarises research data accumulated during the past 10 years. Yet the fact that that particular population suffers from distinct stressors makes it hard for us to compare it with the Israeli population. The only common factors are the fact that the two populations live in the same geographical area and under a permanent existential stress.

Few studies have been conducted in Israel on PTSD reactions to the stress caused by the unstable safety situation. Three of them will be mentioned here, one concerning the general population and the others concerning children from Jerusalem. The results of an original study on PTSD in Israeli adolescents by Dr Pat-H orenczyk and colleagues will be reported at the 19th Annual Meeting of the International So ciety for Traumatic Stress Studies in Chicago in 0 ctober (please contact Dr Pat-Horenczyk for further details: rpat@ herzoghospital.org).

\section{Israeli studies of PTSD}

The study concerning the general population was conducted by Bleich et al (2003), who examined the effect on the Israeli population of media reports of terro rist acts. 0 ver the past two years a total of 4584 people were injured and 622 killed in Israel by such acts. In this study, the degree of exposure to stress and the frequency of PTSD were measured among a representative sample of the general population in Israel. The authors found that $44 \%$ of the sample were personally involved or had at least one relative who was exposed to a terrorist attack. This is a very high degree of exposure, in fact one of the highest described in the literature. Although only $9.4 \%$ of the sample presented full-blown PTSD, as much as $55 \%$ had at least one symptom of post-traumatic stress. N onetheless, $82 \%$ of the sample reported feeling optimistic about their personal future. The authors also gave an overview of the coping mechanisms used by the sample. They concluded that the surprisingly moderate psychopathological impact of the violence was primarily due to a psychological process of adaptation to living in such a stressful environment, and indicates the degree of resilience of Israeli society.

0 ne of the most interesting studies, in my opinion, concerning PTSD in children has been conducted by Lavi $\&$ Solomon (Solomon, personal communication, 2003), from Tel Aviv U niversity, who examined exposure rates and PTSD prevalence rates among children from six different populations: from the centre of Jerusalem, G iloh (a suburb of Jerusalem which has suffered many casualties), Efrat (a residential area within the occupied
The surprisingly moderate psychopathological impact of the violence was primarily due to a psychological process of adaptation to living in such a stressful environment, and indicates the degree of resilience of Israeli society. 
Researchers still differ on their definitions of PTSD, in the assessment tools they use and on the correlations they find.

\section{Common general} findings concerning the reaction to prolonged

stressors have

been found, such as the fact that the degree of exposure is not directly related to PTSD morbidity. The next step is to analyse environmental factors in order to detect what protective factors are responsible for this. territories), Katif (a settlement in the Gaza Strip), from the Palestinian Authority, and Israeli-Arab children.

It is noteworthy that the authors insisted on using the term examine, and not comparing: they believe that the results obtained from the different populations are not comparable, due to differences in degrees of exposure to combat and terror, differences in culture, and differences in socio-economic status. The authors also indicated that such comparisons could raise ethical objections.

Exposure was estimated by the number of lifethreatening events that the children had experienced. Exposure rates (\%) were: Katif, 11.6; Palestinian Authority, 10.0; Efrat, 8.5; Israeli-Arabs, 6.9; G iloh, 3.4; centre of Jerusalem, 2.8. Boys, in general, had been more often exposed than girls. Evidence for PTSD was found among $70.2 \%$ of the Palestinian population and $50.2 \%$ of the Israeli-Arab population. According to the authors, these results are similar to those reported by Saigh (1991) in his study in Beirut and Kuw ait. Concerning the Jewish population, the proportions with a PTSD diagnosis were as follows: Katif, 27.9\%; Efrat, $27.4 \%$; G iloh, 16.4\%; centre of Jerusalem, $13.9 \%$. These results indicate that children who live in the territories show the same prevalence of PTSD as the population living in war zones. Finally, the authors attempted to link personal, religious, cultural and ideological beliefs with PTSD symptoms.

Finally, in June and July of this year, D rs Galili-Weisstub and Ben-Harosh, from the Hadassah Hebrew Medical School in Jerusalem, examined post-traumatic symptoms among 163 minors who were brought to the emergency room of $\mathrm{H}$ adassah H ospital, atter 41 terro rist attacks. This sample consisted of 32 children (20\%) and 131 adolescents ( $80 \%$ ). 0 f the sample, $23.3 \%$ had to be admitted (three of whom died); the remaining $76.7 \%$ were discharged. Examination revealed that $42.9 \%$ of the children had the PTSD symptoms of re-experiencing, avoidance or hyperarousal (of whom $45.7 \%$ were boys and $54.2 \%$ girls) (G alili-Weisstub, personal communication, 2003).

\section{Conclusions}

We should be very cautious to day. We are not able yet to compare research results, unless similar samples and tools are used. At the moment, researchers still differ on their definitions of PTSD, in the assessment tools they use and on the correlations they find with other variables. $\mathrm{N}$ evertheless, common general findings concerning the reaction to prolonged stressors have been found, such as the fact that the degree of exposure is not directly related to PTSD morbidity. The next step is to analyse environmental factors in order to detect what protective factors are responsible for this.

Given the widespread morbidity of PTSD, we should start to think in terms of 'mass trauma', which leads us to consider 'mass treatments'. We should seek ways to use the mass media to instil resiliency in the general population. $H$ istory and some form of slow 'immunisation' may be the leading horizontal factors underlying resiliency. It is important for the scientific community to find out what are the vertical, actual and practical protective components, perhaps hope and faith, which can be used both in our generation and in order to immunise the next generations from this debilitating psychopathology.

\section{References}

Bleich, A., Gelkopf, M. \& Solomon, Z. (2003) Exposure to terrorism, stress-related mental health symptoms and coping behaviors among a nationally representative sample in Israel. JAM A, 290, 612-620.

Saigh, P. A. (1991) O $n$ the development of PTSD following four different types of traumatization. Behaviour Research \& Therapy, 29, 213-216.

\title{
Neither war nor peace: children and youth in organised armed violence
}

\author{
Luke Dowdney
}

Viva Rio / ISER (Instituto de Estudos da Religião), Ladeira da Glória, 98, Glória, CEP: 22211-120, Rio de Janeiro -

RJ, Brazil, email luke@vivario.org.br

he international community has been slow to appreciate the growing problem of the participation of armed children and youths in nonpolitical disputes, encountered in both developed and developing countries, from $\mathrm{H}$ aiti to $\mathrm{N}$ orthern Ireland. While there is widespread recognition of the issue of 'child soldiers' (e.g. www.childsoldiers.org/) there are also many children who participate in organised armed groups that function outside traditionally defined war zones. N owhere 